\title{
Performance Limitations for Linear Feedback Systems in the Presence of Plant Uncertainty
}

\author{
Graham C. Goodwin, Mario E. Salgado, and Juan I. Yuz
}

\begin{abstract}
The goal of this paper is to contribute to the understanding of fundamental performance limits for feedback control systems. In the literature to date on this topic, all available results assume that the designer has an exact model of the plant. Heuristically, however, one would expect that plant uncertainty should play a significant role in determining the best achievable performance. The goal of this paper is to investigate performance limitations for linear feedback control systems in the presence of plant uncertainty. We formulate the problem by utilizing stochastic embedding of the uncertainty. The results allow one to evaluate the best average performance in the presence of uncertainty. They also allow one to judge whether uncertainty or other properties, e.g., nonminimum phase behavior, are dominant limiting factors.
\end{abstract}

Index Terms-Performance limitations, stochastic embedding, uncertainty.

\section{INTRODUCTION}

$\mathbf{F}$ UNDAMENTAL limitations on the performance of feedback control loops have been a topic of interest since the seminal work of Bode during the 1940s related to feedback amplifier design [1]. There are several well-known examples where one can readily appreciate the link between structure and the associated limits on control-loop performance; see, for example, the discussion of the inverted pendulum [2] or the flight controller for the X-29 aircraft [3].

The tools for analyzing limits of performance for systems without uncertainty include logarithmic sensitivity integrals, limiting quadratic optimal control and entropy measures. Early work focused on linear feedback systems; see, for example, [1]-[12]. There has also been growing interest in performance limitations for nonlinear feedback systems; see, for example, [13]-[15].

To give a flavor of the results achieved to date, we note that one can distinguish two types of performance constraints, namely:

i) those that hold for all designs, independent of the criterion used to design the controller;

ii) those that hold for a best design, based upon some given optimality criterion.

Examples of fundamental limitations of type i) are the Bode-Poisson integral formulas for a control loop as in Fig. 1,

Manuscript received March 30, 2002; revised November 19, 2002. Recommended by Guest Editor R. H. Middleton.

G. C. Goodwin and J. I. Yuz are with the School of Electrical Engineering and Computer Science, The University of Newcastle, Newcastle, NSW 2308, Australia (e-mail: eegcg@ee.newcastle.edu.au; jiyuze@ee.newcastle.edu.au).

M. E. Salgado is with Departamento de Electrónica, Universidad Técnica Federico Santa María, Casilla 110-V, Valparaíso, Chile (e-mail: mario.salgado@elo.utfsm.cl).

Digital Object Identifier 10.1109/TAC.2003.815011

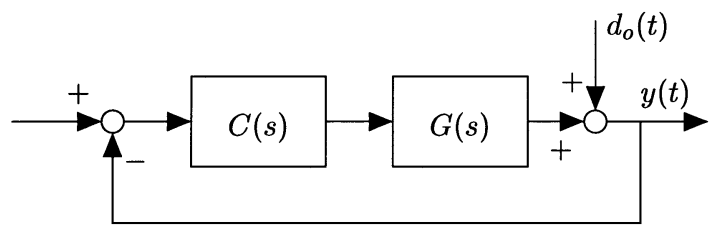

Fig. 1. Feedback control loop.

where the plant and the controller are linear. For example, in the single-input-single-output (SISO) case, the achieved complementary sensitivity is known to satisfy an integral constraint depending on open right-half plane (ORHP) zeros and time delays. Similar, the achieved sensitivity function is known to satisfy an integral constraint depending on ORHP poles.

Well known examples of the type ii) limitations are best $\mathcal{L}_{2}$ cheap control. These give the minimum achievable integral square output error due to unit step output disturbances and/or impulsive measurement noise.

These bounds can be established via frequency domain arguments [16], [2], [10], and can be extended to plants which are both nonminimum phase or unstable [6]. There also exist interesting connections between the cheap control results and the Bode-Poisson integral equations [17].

A key point here is that results of type i) hold for all stabilizing controllers, whereas bounds of type ii) require that a very specific control law be used and they are thus sensitive to the fidelity of the model used for design purposes. Based on this observation, the goal of the current paper is to quantify the impact of model uncertainty on the best achievable performance of a control loop. Our analysis is in the spirit of the questions posed in [18] in a general nonlinear setting. Here, we consider a simple linear feedback system so as to maximally benefit from insight and so as to retain the spirit of the usual performance limits when the plant model is known [2], [6]. We consider SISO control loops for open-loop stable plants and focus on best $\mathcal{L}_{2}$ performance with step disturbance rejection.

\section{Performance Limits With No Model UnCertainty}

As a precursor to the subsequent work on limits with plant uncertainty, we will first present the corresponding results when the plant model is known exactly.

We consider an open-loop stable linear model $G_{o}(s)$ and an output disturbance $d_{o}(t)=\mu(t)$, i.e., a unit step. We assume that $G_{o}(s)$ can be written as

$$
G_{o}(s)=e^{-s \tau_{o}} B_{T}(s) \tilde{G}_{o}(s)
$$


where

$\tau_{o}$ is the pure time delay of the plant

$B_{T}(s)=\prod_{i=1}^{N_{c}} \frac{-s+c_{i}}{s+c_{i}} ; \quad \operatorname{Re}\left(c_{i}\right)>0$

$\tilde{G}_{o}(s)$ is stable, rational, minimum phase, and

$\lim _{s \rightarrow 0} \tilde{G}_{o}(s)=k \neq 0$.

We do not restrict ourselves to linear feedback control. Instead, we consider any input $u(t), t \in[0, \infty$ ), (no matter how generated) satisfying the following two conditions.

i) $u(t)$ is such that its Laplace transform, $U(s)$, is well defined, and is such that $s U(s)$ is analytic in the right-half plane (RHP).

ii) $u(t)$ has a constant component which asymptotically compensates the disturbance, i.e., takes $y(\infty) \rightarrow 0$.

We then have the following result (which extends the results in [5] and [6] to cover general inputs and plants having pure time delays).

Theorem 1: Given a perfectly known stable linear SISO plant having transfer function as in (1) and any input satisfying conditions i) and ii), then, for a unit step output disturbance, the minimum $\mathcal{L}_{2}$ norm of the output satisfies

$$
\min \left\{\int_{0}^{\infty} y^{2}(t) d t\right\}=\tau_{o}+2 \sum_{\ell=1}^{N_{c}} \frac{1}{c_{\ell}} \triangleq J_{\text {cert }}
$$

where $\tau_{o}$ is the pure time delay, and $\left\{c_{1}, \ldots, c_{N_{c}}\right\}$ are the ORHP zeros of the plant.

Proof: See Appendix A.

Remark 1: The above result is well known in the performance limitation literature. However, a key point in this paper is that the bound on the right-hand side of (2) requires that the optimal input be expressed as a function of the true system model [2], [6]. The performance of controllers designed to achieve the limiting performance (2) is well known to be very sensitive to the fidelity of the plant model (see Section VI-B). Indeed, the linear feedback form of the result is rendered unstable by arbitrarily small undermodeling. We are thus motivated to examine the impact of undermodeling on the achievable performance. This topic will be addressed in Section IV. As a prelude, in the following section, we will introduce the class of undermodeling that we will utilize.

\section{DESCRIPTION OF A ClASS OF MODEL ERRORS}

In view of the comments made at the end of the previous section, we proceed to consider the impact of modeling errors. In particular, we assume that

$$
G_{\epsilon}(s)=G(s)-G_{o}(s)=G_{o}(s) G_{\Delta}(s)
$$

where $G_{\epsilon}(s)$ and $G_{\Delta}(s)$ are the additive and relative model error, respectively.

Typically the fidelity of the nominal model used to represent a plant will deteriorate at higher frequencies. To illustrate this we analyze three typical examples. i) Unmodeled delay and d.c. gain error

$$
G=\left(1+K_{u}\right) e^{-s T_{u}} G_{o} ; \quad G_{\Delta}=\left(1+K_{u}\right) e^{-s T_{u}}-1
$$

ii) Unmodeled pole and d.c. gain error

$$
G=\frac{\left(1+K_{u}\right)}{s T_{u}+1} G_{o} ; \quad G_{\Delta}=\frac{\left(1+K_{u}\right)}{s T_{u}+1}-1 .
$$

iii) Unmodeled zero and d.c. gain error

$$
G=\left(1+K_{u}\right)\left(s T_{u}+1\right) G_{o} ; \quad G_{\Delta}=\left(1+K_{u}\right) T_{u} s+K_{u}
$$

In each case, it is readily seen that the relative model error can be bounded as follows:

$$
\left|G_{\Delta}(j \omega)\right| \leq|\alpha|+|\omega \beta|
$$

where $\beta=T_{u}$ for cases i) and ii), $\beta=\left(1+K_{u}\right) T_{u}$ in case iii), and $\alpha=K_{u}$ in each of the cases.

For the previous examples, we have a common bound on the magnitude of $\left|G_{\Delta}(j \omega)\right|$, which is a constant plus a term which grows linearly with frequency. We will capture these features of modeling errors using the idea of stochastic embedding [19], [20]. The core idea is to think of the given modeling error as a particular realization of a stochastic process in the frequency domain. Thus, we write

$$
G_{\Delta}(s)=G_{\Delta}^{0}+s G_{\Delta}^{1}
$$

where $G_{\Delta}^{0}$ and $G_{\Delta}^{1}$ are independent random variables such that $E\left\{G_{\Delta}^{0}\right\}=0, E\left\{G_{\Delta}^{1}\right\}=0$, and

$$
\begin{aligned}
E\left\{G_{\Delta}(j \omega)\right\} & =E\left\{G_{\Delta}^{0}+j \omega G_{\Delta}^{1}\right\}=0 \\
E\left\{\left|G_{\Delta}(j \omega)\right|^{2}\right\} & =E\left\{\left|G_{\Delta}^{0}\right|^{2}\right\}+\omega^{2} E\left\{\left|G_{\Delta}^{1}\right|^{2}\right\}=\frac{1}{a^{2}}+\frac{\omega^{2}}{b^{2}} .
\end{aligned}
$$

The form of these results implies that the mean square modeling error corresponds to the magnitude of the frequency response of a single zero, as shown in Fig. 2. For illustrative purposes, we have reparametrized (10), in terms of the d.c. modeling error $0 \leq d<1$ (more than $100 \%$ would be intolerable) and the parameter $u$, defined as the frequency where $E\left\{\left|G_{\Delta}(j \omega)\right|^{2}\right\}=1$. Note that $d=a^{-1}$ and $u=b \sqrt{1-a^{-2}}$.

In the light of (4)-(7), the mean square result given in (10) may be considered as representative of typically encountered undermodeling scenarios.

We next give a physical interpretation to the constant $u$; specifically, it is the frequency at which the mean square relative uncertainty has reached 1 . Thus, we will call $u$ the model certainty bandwidth (i.e., the range of frequencies until the relative model error reaches $100 \%$ ).

In the next section, we design a fixed control law that minimizes the best average performance for this class of model errors. This result will then be used as to quantify the expected true performance in the presence of model errors. 


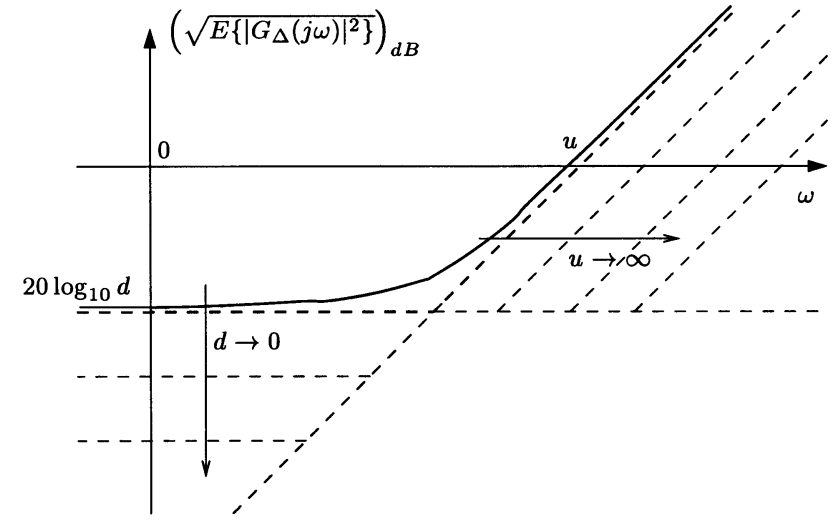

Fig. 2. Modeling error bound.

\section{RoBUST REDESIGN}

In the case of open-loop stable plants with exactly known model, we found that the results in Section II hold for general inputs and are thus not restricted to linear feedback control. However, this will certainly not be the case in the presence of modeling errors since the nature of the feedback plays an important role in the achievable performance. Thus, we will restrict ourselves to linear feedback control laws which stabilize the nominal plant. (This being the minimal requirement to obtain a meaningful performance bound).

Thus consider the linear feedback control loop shown in Fig. 1. As before, we are interested in the minimum value for

$$
J=\int_{0}^{\infty} y^{2}(t) d t=\frac{1}{2 \pi} \int_{-\infty}^{\infty}|Y(j \omega)|^{2} d \omega=\|Y(j \omega)\|_{2}^{2} .
$$

Since the plant is assumed to be open-loop stable, the Youla parametrization of all stabilizing controllers for the nominal plant can be written in the form [16]

$$
C(s)=\frac{Q(s)}{1-G_{o}(s) Q(s)}
$$

where $Q(s)$ is a proper, stable transfer function. Based on this parametrization, the nominal sensitivity functions are given by

$$
\begin{aligned}
& T_{o}(s)=G_{o}(s) Q(s) \\
& S_{o}(s)=1-T_{o}(s)=1-G_{o}(s) Q(s) .
\end{aligned}
$$

Notice that the result of Section II is obtained with the particular linear feedback control law $Q(s)=-s U(s)$, with $U(s)$ as in $(50)$ and $\tilde{U}(s)=0$.

Of course, with modeling errors, the nominal sensitivities given in (13) and (14) will not be realized. Instead, the achieved (i.e., true) sensitivity function will be given by [16, p. 410]

$$
S_{1}(s)=\frac{1-G_{o}(s) Q_{1}(s)}{1+G_{o}(s) Q_{1}(s) G_{\Delta}(s)}
$$

where $G_{\Delta}(s)$ is the relative model error as in (3) and $Q_{1}(s)$ is some new value for the parameter in (12). In particular, our goal will be to redesign the optimal nominal controller, $Q_{o}(s)$, so as to best cope, in an average sense, with the assumed class of model errors.
In the same spirit as the use of relative model errors in (3), we will find it convenient to utilize relative changes in $Q(s)$. Thus, we write the redesigned $Q(s)$ parameter in terms of $Q_{o}(s)$ using

$$
Q_{1}(s)=Q_{o}(s)\left(1+Q_{\Delta}(s)\right) .
$$

Note that $Q_{\Delta}(s)$ must be zero at d.c. to preserve integral action. Thus, in the sequel, we will assume that $Q_{\Delta}(s)$ has the form

$$
Q_{\Delta}(s)=s Q_{\Delta}^{\prime}(s) .
$$

Substituting into (15), we have

$$
S_{1}(s)=\frac{S_{o}(s)-T_{o}(s) Q_{\Delta}(s)}{1+T_{o}(s)\left(1+Q_{\Delta}(s)\right) G_{\Delta}(s) .}
$$

Since we are considering a class of random modeling errors with properties as in (9)-(10), we replace the deterministic cost function (11) by the average performance over the assumed class of model errors. Thus, we consider

$$
\bar{J}=E\left\{\int_{0}^{\infty} y(t)^{2} d t\right\}
$$

where the expectation is over the (stochastic) class of model errors. Thus, for each realization of the model error, we have that

$$
Y(s)=\frac{S_{1}(s)}{s}
$$

where $S_{1}(s)$ is a random variable as in (18) and $G_{\Delta}(s)$ is a particular realization of the undermodeling drawn from a distribution satisfying (9)-(10).

Again, using (20) and Parseval's theorem, the average performance over the assumed class of model errors is

$$
\begin{aligned}
\bar{J} & =E\left\{\frac{1}{2 \pi} \int_{-\infty}^{\infty} \frac{\left|S_{1}(j \omega)\right|^{2}}{\omega^{2}} d \omega\right\} \\
& =\frac{1}{2 \pi} \int_{-\infty}^{\infty} \frac{E\left\{\left|S_{1}(j \omega)\right|^{2}\right\}}{\omega^{2}} d \omega
\end{aligned}
$$

where we assume sufficient regularity to interchange the integral and expectation operators.

Our aim in the next section will be determine a fixed $Q_{\Delta}(s)$ that optimizes (21). With this redesigned control law, we will then evaluate the associated expected performance over the given model error class.

We note that the right-hand side of (18) is a nonlinear function of $Q_{\Delta}(s)$ and $G_{\Delta}(s)$. This would render any attempt to carry out an optimization of expected performance intractable. However, closed-loop stability requires that $\left|\left(1+Q_{\Delta}\right) G_{\Delta}\right|$ be less than 1 . Thus, we make a Taylor's series expansion in $(1+$ $\left.Q_{\Delta}\right) G_{\Delta}$ as follows:

$$
\begin{aligned}
S_{1} \approx & {\left[S_{o}-T_{o} Q_{\Delta}\right]\left[1-T_{o}\left(1+Q_{\Delta}\right) G_{\Delta}\right] } \\
= & S_{o}-T_{o} Q_{\Delta}-S_{o} T_{o}\left(1+Q_{\Delta}\right) G_{\Delta} \\
& +T_{o}^{2}\left(1+Q_{\Delta}\right) Q_{\Delta} G_{\Delta} .
\end{aligned}
$$

The last term in the previous expression depends in the product of $\left(1+Q_{\Delta}\right), Q_{\Delta}$, and $G_{\Delta}$. At those frequencies where $\left|G_{\Delta}\right|$ is small, then $\left|Q_{\Delta}\right|$ will also be small (i.e., there is no need 
to change the nominal controller). At those frequencies where $\left|G_{\Delta}\right|$ approaches 1 , then to preserve stability, it is necessary that $Q_{1}$ go to zero, i.e., $\left(1+Q_{\Delta}\right)$ should approach zero. We may thus argue that the combination of factors in the last term previously shown ensures that this term should be small relative to the others. Thus, we further approximate (22) by

$$
S_{1} \approx\left(S_{o}-T_{o} Q_{\Delta}\right)-S_{o} T_{o}\left(1+Q_{\Delta}\right) G_{\Delta} .
$$

Substituting the approximation (23) into (21) and utilizing (9)-(10) we obtain

$$
\begin{aligned}
\bar{J} \approx \frac{1}{2 \pi} \int_{-\infty}^{\infty} \frac{\left|S_{o}-T_{o} Q_{\Delta}\right|^{2}}{\omega^{2}} & \\
& +\frac{\left|S_{o}\right|^{2}\left|T_{o}\right|^{2}\left|1+Q_{\Delta}\right|^{2}\left(\omega^{2}+\frac{b^{2}}{a^{2}}\right)}{b^{2} \omega^{2}} d \omega \triangleq \bar{J}^{\prime} .
\end{aligned}
$$

Equation (24) will be the basis of the analysis presented below. We will return to the approximations involved in deriving (24) in Section VI.

\section{Limiting Average PeRformance}

For clarity of exposition, we begin with the case of one nonminimum phase zero in the nominal model. We will later extend this to multiple nonminimum phase zeros and/or delays.

\section{A. Single Nonminimum Phase Zero in the Nominal Plant}

Suppose that the nominal model of the plant has one nonminimum phase zero and no pure time delay. We can then write

$$
G_{o}(s)=\frac{-s+c}{s+c} \tilde{G}_{o}(s)=B_{T}(s) \tilde{G}_{o}(s)
$$

where $c>0$ and $\tilde{G}_{o}(s)$ is rational and minimum phase.

The optimal nominal design is given by

$$
Q_{o}(s)=\left[\tilde{G}_{o}(s)\right]^{-1} .
$$

Substituting $T_{o}(s)=T_{o}^{*}(s)=B_{T}(s)$ and $S_{o}(s)=S_{o}^{*}(s)=$ $1-B_{T}(s)$ into (24) and making use of (17), we see that $\bar{J}^{\prime}$ becomes $\bar{J}$, where

$$
\begin{aligned}
\bar{J}=\frac{1}{2 \pi} \int_{-\infty}^{\infty} \frac{\left|2-(-j \omega+c) Q_{\Delta}^{\prime}\right|^{2}}{\mid j \omega} & \\
& +\frac{4|1+c|^{2}}{b^{2}\left|j \omega+Q_{\Delta}^{\prime}\right|^{2}\left|j \omega+\frac{b}{a}\right|^{2}} d \omega .
\end{aligned}
$$

Theorem 2: We then have the following results.

i) The average cost function $\overline{\bar{J}}$ is minimized by the choice

$$
Q_{\Delta_{\mathrm{opt}}}^{\prime}(s)=\frac{-\left(2 s+b \sqrt{1+\frac{4}{a^{2}}+\frac{4 c}{b}}-b\right)}{2 s^{2}+b \sqrt{1+\frac{4}{a^{2}}+\frac{4 c}{b}} s+b c} .
$$

ii) The resulting best average performance is given by

$$
\overline{\bar{J}}_{\mathrm{opt}}=\frac{2}{c}+\frac{1}{c}\left(-1+\sqrt{1+\frac{4}{a^{2}}+\frac{4 c}{b}}\right) .
$$

Proof: We follow the procedure outlined, for example, in [16, p. 467]. Specifically, we rewrite $\overline{\bar{J}}$ as

$$
\begin{aligned}
\bar{J}=\frac{1}{2 \pi} \int_{-\infty}^{\infty} \mid & Q_{\Delta}^{\prime}(j \omega) H(j \omega)+\left.\frac{F(j \omega)}{H(-j \omega)}\right|^{2} \\
& +\frac{4\left(b^{2}+\left|j \omega+\frac{b}{a}\right|^{2}\right)}{b^{2}|j \omega+c|^{2}}-\left|\frac{F(j \omega)}{H(-j \omega)}\right|^{2} d \omega
\end{aligned}
$$

where $H(j \omega)$ and $F(j \omega)$ are obtained from (27). In particular, $H(j \omega)$ is obtained by spectral factorization.

Note that the minimum of (30) depends only on the first term and is achieved by making the choice

$$
Q_{\Delta}^{\prime}(s)=-\frac{1}{H(s)}\left\{\frac{-2 b^{2}(s+c)-4 s\left(-s^{2}+\frac{b^{2}}{a^{2}}\right)^{3}}{b^{2}\left(-s^{2}+c^{2}\right) H(-s)}\right\}_{\substack{\text { stable } \\ \text { part }}}
$$

which gives (28), establishing i). Note that the redesigned controller is easily obtained from (16) and (17). Result ii) is obtained by substituting the optimal value of $Q_{\Delta}^{\prime}(s)$ into (27), and evaluating the integral via residues.

Remark 2: It is important to note that when uncertainty is negligible, i.e., both parameters $a$ and $b$ grow to infinity, i.e., there is no uncertainty in the d.c. gain $d \rightarrow 0$ and the certainty bandwidth $u$ satisfies $u \rightarrow \infty$, the performance bound obtained in Theorem 2 reduces to the one given in Theorem 1.

Remark 3: We can always scale the result by $c$ since this simply amounts to redefining the units of time. Fig. 3 shows the corresponding scaled value of the bound (29) as a function of the dimensionless quantity $d$ and the (scaled) uncertainty parameter $c / u$.

Remark 4: It is difficult to treat exactly a pure time delay within the given framework since this requires the spectral factorization of a transcendental function. However, a guide to the achieved robust performance with a pure time delay in the nominal model can be obtained by use of a first-order Padé approximation [16]

$$
e^{-s \tau_{o}} \approx \frac{1-\frac{s \tau_{o}}{2}}{1+\frac{s \tau_{o}}{2}}
$$

We then obtain the result as in Theorem 2 with $c=2 / \tau_{o}$ or specifically

$$
\overline{\bar{J}}_{\mathrm{opt}}=\tau_{o}+\frac{\tau_{o}}{2}\left(-1+\sqrt{1+\frac{4}{a^{2}}+\frac{8}{b \tau_{o}}}\right) .
$$

Again, we notice that this reduces to the result in Theorem 1 when the parameters $a$ and $b$ grow to infinity.

\section{B. More General Nominal Plants}

The same basic procedure as exemplified in Section V-A can be used for general nominal plants. One can always obtain numerical bounds using this methodology. However, in the spirit of Theorem 2, it is also desirable to obtain analytical expressions since these give additional insight into the impact of the various factors on the performance limit. The expressions become more complex as the nominal model complexity increases. To illustrate the principle, we consider the case of two nonminimum 


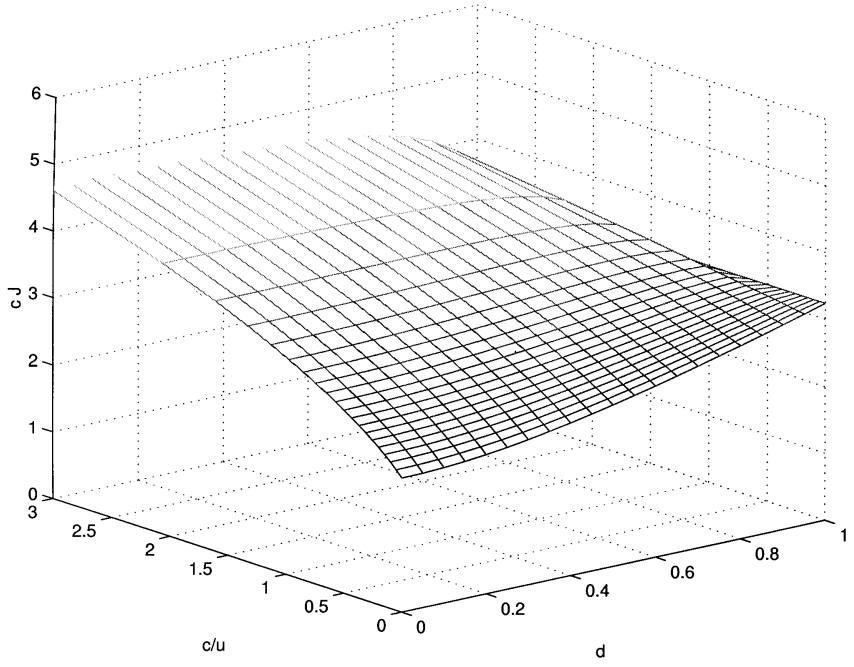

Fig. 3. Bound for one nonminimum phase zero $\mathrm{v} / \mathrm{s}(d, c / u)$.

phase zeros $c_{1}$ and $c_{2}$ in the nominal plant [or, equivalent, one nonminimum phase zero and a pure time delay approximated as in (32)]. We then have the following theorem.

Theorem 3: (Subject to the previous conditions).

i) The cost function $\bar{J}^{\prime}$ is minimized by the choice

$$
Q_{\Delta_{\mathrm{opt}}}^{\prime}(s)=\frac{\left(s+c_{2}\right) A+\left(s+c_{1}\right) B}{\alpha\left(s+z_{1}\right)\left(s+z_{2}\right)}
$$

where

$$
\begin{aligned}
\alpha= & \sqrt{1+\frac{4\left(c_{1}+c_{2}\right)^{2}}{b^{2}}} \\
A= & \frac{4 c_{1}\left(c_{1}+c_{2}\right)^{2}\left(c_{1}^{2}+b^{2}\right)}{\alpha a^{2} b^{2}\left(c_{1}+z_{1}\right)\left(c_{1}+z_{2}\right)\left(-c_{1}+c_{2}\right)} \\
B= & \frac{-4 c_{2}\left(c_{1}+c_{2}\right)^{2}\left(c_{2}^{2}+b^{2}\right)}{\alpha a^{2} b^{2}\left(c_{2}+z_{1}\right)\left(c_{2}+z_{2}\right)\left(-c_{1}+c_{2}\right)} \\
z_{1}= & \frac{\sqrt{c_{1}^{2}+c_{2}^{2}}}{\alpha}\left(\sqrt{1+\frac{4\left(c_{1}+c_{2}\right)^{2}}{a^{2}\left(c_{1}^{2}+c_{2}^{2}\right)}+\frac{2 \alpha c_{1} c_{2}}{\left(c_{1}^{2}+c_{2}^{2}\right)}}\right. \\
& \left.+\sqrt{1+\frac{4\left(c_{1}+c_{2}\right)^{2}}{a^{2}\left(c_{1}^{2}+c_{2}^{2}\right)}-\frac{2 \alpha c_{1} c_{2}}{\left(c_{1}^{2}+c_{2}^{2}\right)}}\right) \\
z_{2}= & \frac{\sqrt{c_{1}^{2}+c_{2}^{2}}}{\alpha}\left(\sqrt{1+\frac{4\left(c_{1}+c_{2}\right)^{2}}{a^{2}\left(c_{1}^{2}+c_{2}^{2}\right)}+\frac{2 \alpha c_{1} c_{2}}{\left(c_{1}^{2}+c_{2}^{2}\right)}}\right. \\
& \left.-\sqrt{1+\frac{4\left(c_{1}+c_{2}\right)^{2}}{a^{2}\left(c_{1}^{2}+c_{2}^{2}\right)}-\frac{2 \alpha c_{1} c_{2}}{\left(c_{1}^{2}+c_{2}^{2}\right)}}\right) .
\end{aligned}
$$

ii) The resulting average performance is given by

$$
\overline{\bar{J}}_{\mathrm{opt}}=2\left(\frac{1}{c_{1}}+\frac{1}{c_{2}}\right)+\tilde{J}\left(c_{1}, c_{2}, a, b\right)
$$

where $\tilde{J}\left(c_{1}, c_{2}, a, b\right)$ is a function such that

$$
\lim _{\substack{a \rightarrow \infty \\ b \rightarrow \infty}} \tilde{J}\left(c_{1}, c_{2}, a, b\right)=0 .
$$

Proof: The proof follows exactly the same steps in Theorem 2. Note that we have not spelled out the form of $\tilde{J}\left(c_{1}, c_{2}, a, b\right)$ due to its complexity. The result is available from the authors on request.

Remark 5: Again we note that (40), agrees with Theorem 1 when the uncertainty is small, i.e., when $a$ and $b$ go to infinity. Specifically, we have that

1) the result obtained agrees with (2), considering two non minimum phase zeros and no nominal time delay $\left(\tau_{o}=\right.$ $0)$;

2) it also agrees with the same result, considering only one non minimum phase zero at $s=c_{1}$ and a pure time delay $\tau_{o}$, approximated by $c_{2}=(2) /\left(\tau_{o}\right)$.

\section{Simulation Results}

To further illustrate the results, we will consider a stable, firstorder plant having a pure time delay and nominal model given by

$$
G_{o}(s)=\frac{e^{-s}}{s+1} .
$$

The optimal nominal controller, using (26), is

$$
Q_{o}(s)=\frac{s+1}{\beta s+1}, \quad \text { where } \beta \rightarrow 0 .
$$

If there is no uncertainty in the plant model, the best achievable performance (when a unit step output disturbance is injected in the control loop) is given by Theorem 1, i.e., $J_{\text {cert }}=1$.

Next, we will suppose that there is uncertainty in the nominal plant (41) and that the true model takes the form

$$
G(s)=\left(1+K_{u}\right) \frac{e^{-s\left(1+T_{u}\right)}}{s+1} .
$$

We suppose that $K_{u}$ and $T_{u}$ are uniformly distributed and independent random variables in the interval $[-p,+p]$, i.e., the true delay and the true d.c. gain of the plant go from $(1-p)$ to $(1+p)$. Thus, we see that the variance of the random variables $K_{u}$ and $T_{u}$ are given by

$$
E\left\{K_{u}^{2}\right\}=\frac{1}{2 p} \int_{-p}^{p} K_{u}^{2} d K_{u}=\frac{p^{2}}{3}=E\left\{T_{u}^{2}\right\} .
$$

Based on (4) and (10), we take

$$
\frac{1}{a}=\frac{1}{b}=\frac{p}{\sqrt{3}}=0.58 p .
$$

Fig. 4 shows the achieved mean value of $\left|G_{\Delta}(j \omega)\right|^{2}$, considering $p=0.5$ and 100 different values of $K_{u}$ and $T_{u}$, compared with the expression (10). In fact, we can see that this is a very good prediction of the achieved expected uncertainty at least in the frequency range where $\left|G_{\Delta}(j \omega)\right|$ is less than $100 \%$.

\section{A. Optimal Average Performance}

The redesigned controller accounting for plant uncertainty is given by Theorem 2, where we use a Padé approximation for 


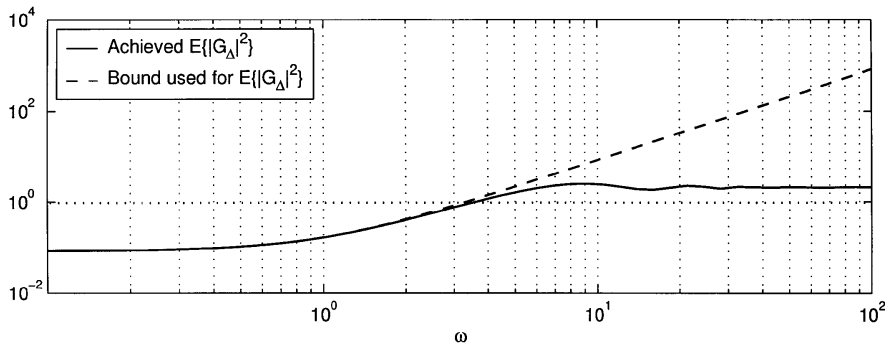

Fig. 4. Modeling error compared with the bound used.

the time delay. Substituting $c=2$ and the variances given in (45) into (28), we obtain

$$
Q_{\mathrm{opt}}(s)=\frac{s+1}{\beta s+1}\left[\frac{s+2}{1.16 p s^{2}+s \sqrt{1+\frac{p^{2}}{3}+4.64 p}+2}\right] .
$$

Fig. 5 shows the actual achieved performance (obtained by simulation and numerical integration of the output from $t=0$ to 50) for different deterministic values of $K_{u}$ and $T_{u}$ in the range $-p$ to $p$ (100 different values for each variable), with $p=0.5$.

The mean value of the achieved performance obtained from the simulations is 1.5363 , and the expected performance bound as predicted by Theorem 2 [in particular, given by (33)] is 1.4543. We see in the figure that there is range of values for $\left(K_{u}, T_{u}\right)$ where the achieved performance is better (less) than the expected performance, and there is another range where the performance is worse (greater).

The average of the achieved performances is only slightly bigger than the average cost predicted by Theorem 2. This is quite remarkable given the various approximations used to develop the result. This vindicates (at least by example) the various approximations used in the derivation of the result.

\section{B. Illustrative Time Responses}

We next illustrate the kind of time response obtained with the nominal controller and the redesigned controller. We consider the same nominal model as in Subsection VI-A save that now we consider a single realization of the uncertainty, where $K_{u}=T_{u}=0.29$. We use the redesigned controller as in (46). Table I shows the time responses of the closed loop systems for the nominal and perturbed plant and for the redesigned control law. Notice that there is a price paid in terms of the nominal performance to achieve robust performance, and, as we expect, the optimal nominal controller has poor robustness because it was designed to achieve the limiting performance in the absence of uncertainty.

\section{CONCLUSION}

This paper has been concerned with the problem of optimal $\mathcal{L}_{2}$ performance for a linear control loop in the presence of a step output disturbance. A distinctive feature of the analysis presented here is that the impact of a (stochastic) class of model uncertainty has been included. A fixed linear feedback control

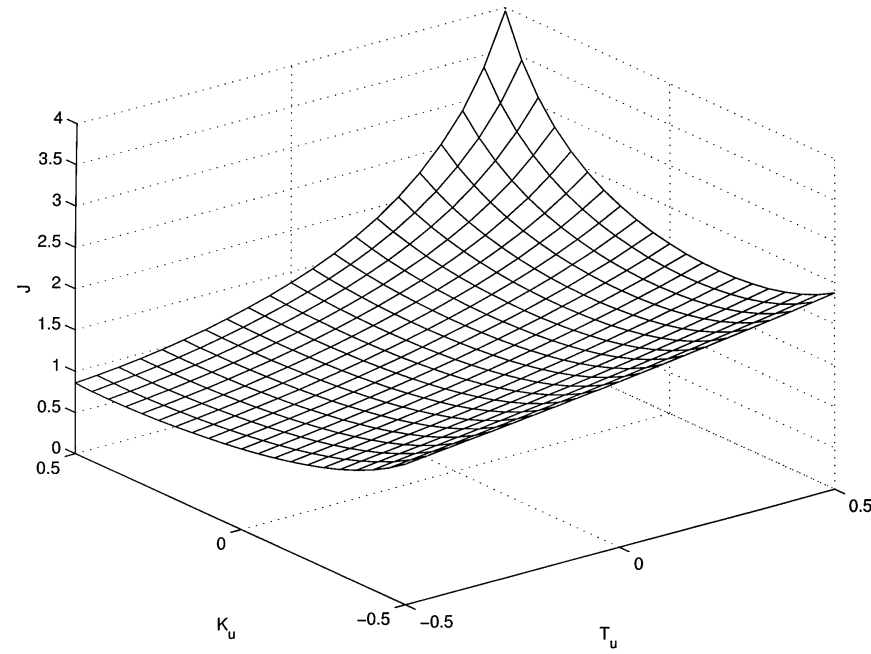

Fig. 5. Achieved performance.

TABLE I

DIFFERENT COMBINATIONS FOR THE PlANT AND CONTROLLER

\begin{tabular}{|c|c|c|}
\hline $\begin{array}{l}\text { Output } \\
y(t)\end{array}$ & $\begin{array}{l}\text { Nominal controller } \\
Q_{o} \quad(\text { with } \beta=0.1)\end{array}$ & $\begin{array}{l}\text { Redesigned controller } \\
Q_{1}=Q_{o}\left(1+Q_{\Delta}\right)\end{array}$ \\
\hline $\begin{array}{l}\text { Nominal } \\
\text { plant: } \\
K_{u}=0 \\
T_{u}=0\end{array}$ & . & \\
\hline $\begin{array}{l}\text { Specific } \\
\text { plant with } \\
\text { uncertainty: } \\
K_{u}=0.29 \\
T_{u}=0.29\end{array}$ & Unstable！ & \\
\hline
\end{tabular}

law has been used with the restriction that this feedback control law must stabilize the nominal system. This does not imply that all plants in the given uncertainty class will necessarily be stabilized. Thus, the results represent a lower bound on the expected performance achievable. The results reduce to the well-known results for the best achievable performance when uncertainty is negligible. The result shows that the average performance in the presence of model uncertainty can depart significantly from best achievable performance without model uncertainty. Thus, the results here are believed to give a more realistic guide to the performance one would expect to achieve in practice.

\section{APPENDIX A}

In this Appendix, we prove Theorem 1. We will make use of the following preliminary result.

Lemma 1: Consider the transfer function

$$
H(s)=\frac{b_{n-1} s^{n-1}+\cdots+b_{1} s+b_{0}}{s^{n}+a_{n-1} s^{n-1}+\cdots+a_{0}}=\frac{\sum_{i=0}^{n-1} b_{i} s^{i}}{\prod_{i=1}^{n_{p}}\left(s-p_{i}\right)^{n_{i}}} .
$$


If $H(s)$ is expanded in its partial fraction decomposition

$$
\begin{aligned}
H(s) & =\frac{\gamma_{1}^{(1)}}{\left(s-p_{1}\right)}+\frac{\gamma_{1}^{(2)}}{\left(s-p_{1}\right)^{2}}+\cdots+\frac{\gamma_{1}^{\left(n_{1}\right)}}{\left(s-p_{1}\right)^{n_{1}}}+\cdots \\
& +\frac{\gamma_{n_{p}}^{(1)}}{\left(s-p_{n_{p}}\right)}+\frac{\gamma_{n_{p}}^{(2)}}{\left(s-p_{n_{p}}\right)^{2}}+\cdots+\frac{\gamma_{n_{p}}^{\left(n_{n_{p}}\right)}}{\left(s-p_{n_{p}}\right)^{n_{n_{p}}}}
\end{aligned}
$$

then

$$
\sum_{\ell=1}^{n_{p}} \gamma_{\ell}^{(1)}=b_{n-1}
$$

Proof: We expand $H(s)$ in powers of $s^{-1}$, i.e., $H(s)=$ $h_{1} s^{-1}+\cdots$. We can see that, if we divide numerator and denominator polynomials in (47), we will obtain $h_{1}=b_{n-1}$.

We now also expand the partial fraction expression (48), dividing every term, where we can see that the coefficient of $s^{-1}$ is $\sum_{\ell=1}^{n_{p}} \gamma_{\ell}^{(1)}$. Hence, (49) is obtained.

Next, we present the proof of Theorem 1.

Proof of Theorem 1: Subject to conditions i) and ii), we can express, without loss of generality, the Laplace transform of the plant input as

$$
U(s)=\frac{-1}{s \tilde{G}_{o}(s)}-\frac{\tilde{U}(s)}{\tilde{G}_{o}(s)}
$$

where $\tilde{U}(s)$ is analytic in the $R H P$ and $\lim _{s \rightarrow \infty} \tilde{U}(s)=0$

The Laplace transform of the plant output is then given by

$$
Y=D_{o}+G_{o} U=\frac{1-B_{T} e^{-s \tau}}{s}-B_{T} e^{-s \tau} \tilde{U} .
$$

We also write $B_{T}(s)=1+s \tilde{B}_{T}(s)$, where $\tilde{B}_{T}(s)$ is stable and strictly proper. Then, from (51), we have that the Fourier transform of the output satisfies

$$
Y=\left(\frac{1-e^{-j \omega \tau}}{j \omega}\right)-e^{-j \omega \tau}\left(\tilde{B}_{T}+\left(1+j \omega \tilde{B}_{T}\right) U\right) .
$$

We note that the first and second terms on the right-hand side are orthogonal. Then, using (11), we have

$$
J=\|Y\|_{2}^{2}=\left\|\frac{1-e^{-j \omega \tau}}{j \omega}\right\|_{2}^{2}+\left\|\tilde{B}_{T}+B_{T} U\right\|_{2}^{2}
$$

Hence, using [16, Lemma 16.2] yields

$$
\begin{aligned}
\operatorname{Arg} \min J & =U_{\mathrm{opt}}=-\left\{B_{T}^{-1}\left[\tilde{B}_{T}\right]\right\}_{\substack{\text { stable } \\
\text { part }}} \\
& =-\left\{B_{T}^{-1}\left[\frac{B_{T}-1}{s}\right]\right\}_{\substack{\text { stable } \\
\text { part }}}=0 .
\end{aligned}
$$

Substituting into (53), and using the Cauchy Integral Theorem [21]

$$
\min J=\tau-\sum_{i=1}^{N} \operatorname{Res}_{s=s_{i}}\left\{\tilde{B}_{T}(s) \tilde{B}_{T}(-s)\right\}
$$

where $\left\{s_{1}, \ldots, s_{N}\right\}$ are the poles of $\tilde{B}_{T}(s) \tilde{B}_{T}(-s)$ lying in the RHP.

We can separate the residues in (55) as follows:

$$
\begin{aligned}
\sum \operatorname{Res}\left\{\tilde{B}_{T}(s) \tilde{B}_{T}(-s)\right\} & =\sum \operatorname{Res}\left\{\frac{1-B_{T}(s)}{-s^{2}}\right\} \\
& +\sum \operatorname{Res}\left\{\frac{1-B_{T}(-s)}{-s^{2}}\right\} .
\end{aligned}
$$

Now, the first term on the right hand side is analytical in the open $R H P$ and hence the residues are zero. Thus we can compute the residues from the residues of the poles of $[1-$ $\left.B_{T}(-s)\right] / s^{2}$ in the ORHP. Now, (57) shown at the bottom of the page holds, where

$$
\alpha=\sum_{\ell=1}^{N_{c}} \frac{\prod_{i=1}^{N_{c}} c_{i}}{c_{\ell}} .
$$

Also, we note that $\left[1-B_{T}(-s)\right] /\left[-s^{2}\right]$ has only one pole at $s=0$. Thus

$$
\frac{1-B_{T}(-s)}{-s^{2}}=\frac{\left[1+(-1)^{N_{c}}\right] s^{N_{c}-1}+\cdots+2 \alpha(-1)^{N_{c}-1}}{-s \prod_{i=1}^{N_{c}}\left(s-c_{i}\right)} .
$$

Hence

$$
\begin{aligned}
& \sum \operatorname{Res}_{\substack{s=s_{i} \\
\operatorname{Re}\left\{s_{i}\right\}>0}}\left\{\tilde{B}_{T}(s) \tilde{B}_{T}(-s)\right\} \\
& =\sum \operatorname{Res}_{\operatorname{Re}\left\{s_{i}\right\}>0}\left\{\frac{1-B_{T}(-s)}{s^{2}}\right\} \\
& =\sum \operatorname{Res}\left\{\frac{1-B_{T}(-s)}{-s^{2}}\right\}-\operatorname{Res}_{s=0}\left\{\frac{1-B_{T}(-s)}{-s^{2}}\right\} \text {. }
\end{aligned}
$$

Then, the residue at $s=0$ is

$$
\begin{aligned}
\operatorname{Res}_{s=0}\left\{\frac{1-B_{T}(-s)}{-s^{2}}\right\} & =\frac{2 \alpha(-1)^{N_{c}-1}}{-(-1)^{N_{c}} \prod_{i=1}^{N_{c}} c_{i}}=\frac{2 \alpha}{\prod_{i=1}^{N_{c}} c_{i}} \\
& =2 \sum_{\ell=1}^{N_{c}} \frac{1}{c_{\ell}}
\end{aligned}
$$

where (58) was used.

Applying Lemma 1, with $n=N_{c}+1$

$$
\sum \operatorname{Res}\left\{\frac{1-B_{T}(-s)}{-s^{2}}\right\}=0
$$

$$
\begin{aligned}
1-B_{T}(-s) & =1-\prod_{i=1}^{N_{c}} \frac{s+c_{i}}{-s+c_{i}}=1-(-1)^{N_{c}} \prod_{i=1}^{N_{c}} \frac{s+c_{i}}{s-c_{i}} \\
& =1+\frac{(-1)^{N_{c}-1}\left\{s^{N_{c}}+\sum_{i=1}^{N_{c}} c_{i} s^{N_{c}-1}+\cdots+\alpha s+\prod_{i=1}^{N_{c}} c_{i}\right\}}{s^{N_{c}}-\sum_{i=1}^{N_{c}} c_{i} s^{N_{c}-1}+\cdots+(-1)^{N_{c}-1} \alpha s+(-1)^{N_{c}} \prod_{i=1}^{N_{c}} c_{i}}
\end{aligned}
$$


Hence

$$
\sum \operatorname{Res}_{\substack{s=s_{i} \\ \operatorname{Re}\left\{s_{i}\right\}>0}}\left\{\tilde{B}_{T}(s) \tilde{B}_{T}(-s)\right\}=-2 \sum_{\ell=1}^{N_{c}} \frac{1}{c_{\ell}} .
$$

Finally, replacing in (55), the result is obtained.

\section{REFERENCES}

[1] H. W. Bode, Network Analysis and Feedback Amplifier Design. New York: Van Nostrand, 1945.

[2] M. M. Serón, J. Braslavsky, and G. Goodwin, Fundamental Limitations in Filtering and Control. New York: Springer-Verlag, 1997.

[3] K. J. Åström, "Fundamental limitations of control system performance," in Communication, Computation, Control and Signal Processing-A Tribute to Thomas Kailath, A. Paulraj, V. Roychowdhury, and C. D. Schaper, Eds. Boston, MA: Kluwer, 1997, pp. 355-363.

[4] J. Freudenberg and D. Looze, "Right half plane poles and zeros and design tradeoffs in feedback systems," IEEE Trans. Automat. Contr., vol. AC-30, pp. 555-565, June 1985.

[5] L. Qiu and E. Davidson, "Performance limitations of nonminimum phase systems in the servomechanism problem," Automatica, vol. 29 no. 2, pp. 337-349, 1993.

[6] J. Chen, L. Qiu, and O. Toker, "Limitations in maximal tracking accuracy," IEEE Trans. Automat. Contr., vol. 45, pp. 326-331, Feb. 2000.

[7] J. Chen, "Logarithmic integrals, interpolation bounds and performance limitations in MIMO feedback systems," IEEE Trans. Automat. Contr., vol. 45, pp. 1098-1115, June 2000.

[8] J. Chen and C. Nett, "Sensitivity integrals for multivariable discrete time systems," Automatica, vol. 31, no. 8, pp. 1113-1124, Aug. 1995.

[9] G. Gómez and G. Goodwin, "Integral constraints on sensitivity vectors for multivariable linear systems," Automatica, vol. 32, no. 4, pp. 499-518, 1996.

[10] R. Middleton, "Trade-offs in linear control systems design," Automatica, vol. 27, no. 2, pp. 281-292, 1991

[11] V. Sule and V. Athani, "Directional sensitivity trade-offs in multivariable feedback systems," Automatica, vol. 27, no. 5, pp. 869-872, 1991.

[12] H. Sung and S. Hara, "Properties of sensitivity and complementary sensitivity functions in single-input single-output digital control systems," Int. J. Control, vol. 48, no. 6, pp. 2429-2439, 1998

[13] M. Serón and G. Goodwin, "Sensitivity limitations in nonlinear feedback control," Syst. Control Lett., vol. 27, pp. 249-254, 1996.

[14] J. Shamma, "Performance limitations in sensitivity reduction for nonlinear plants," Syst. Control Lett., vol. 17, pp. 43-47, 1991.

[15] P. Iglesias, "An analogue of Bode's integral for stable nonlinear systems: Relations to entropy," Proc. of the 40th IEEE Conf. Decision Control, vol. 4, pp. 3419-3420, 2001.

[16] G. Goodwin, S. Graebe, and M. Salgado, Control System Design. Upper Saddle River, NJ: Prentice-Hall, 2001.

[17] R. Middleton and J. Braslavsky, "On the relationship between logarithmic sensitivity integrals and optimal control problems," Proc. 39th IEEE Conf. Decision Control, vol. 5, pp. 4990-4995, 2000

[18] L. Xie and L. Guo, "How much uncertainty can be dealt with by feedback," IEEE Trans. Automat. Contt. , vol. 45, pp. 2203-2217, Dec. 2000.

[19] G. Goodwin and M. Salgado, "A stochastic embedding approach for quantifying uncertainty in the estimation of restricted complexity models," Int. J. Adapt. Control Signal Processing, vol. 3, pp. 333-356, 1989.
[20] G. Goodwin, J. Braslavsky, and M. Serón, "Non-stationary stochastic embedding for transfer function estimation," Automatica, vol. 38, pp. $47-62,2002$.

[21] R. Churchill and J. Brown, Complex Variable and Applications. New York: McGraw-Hill, 1984.

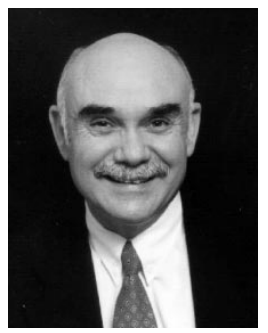

Graham C. Goodwin received the B.Sc. degree in physics, the B.E. degree in electrical engineering, and the Ph.D. degree from the University of New South Wales, New South Wales, Australia, in 1965, 1967, and 1971, respectively.

From 1970 to 1974 , he was a Lecturer in the Department of Computing and Control, Imperial College, London, U.K. Since 1974, he has been with the Department of Electrical and Computer Engineering, The University of Newcastle, Newcastle, NSW, Australia. He is the coauthor of seven monographs, four edited volumes, and several hundred technical papers. He is currently Professor of Electrical Engineering and Associate Director of the Centre for Integrated Dynamics and Control at the University of Newcastle.

Dr. Goodwin was the recipient of several international prizes including the USA Control Systems Society 1999 Hendrik Bode Lecture Prize, a Best Paper award by the IEEE TRANSACTIONS ON AUTOMATIC CONTROL, and a Best Paper Award by the Asian Journal of Control. He was also the recipient of an ARC Federation Fellowship, and he is an Honorary Fellow of the Institute of Engineers, Australia, a Fellow of the Australian Academy of Science, a Fellow of the Australian Academy of Technology, Science, and Engineering, a Member of the International Statistical Institute, and a Fellow of the Royal Society, London.

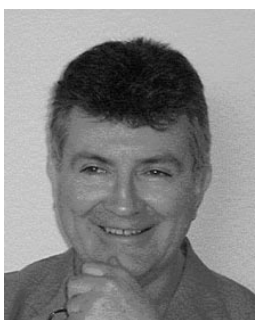

Mario E. Salgado received the professional title of Ingeniero Civil Electrónico from Universidad Técnica Federico Santa María, Valparaíso, Chile, in 1974, the M.Sc. degree from Imperial College, London, U.K., in 1979, and Ph.D. degree in electrical engineering from The University of Newcastle, Newcastle, NSW, Australia, in 1989.

He is currently an Academic with the Department of Electronic Engineering, Universidad Técnica Federico Santa María, where he lectures on linear and control systems at the undergraduate and postgraduate levels. His research areas include control system design and system identification. He coauthored (with G. C. Goodwin and S. F. Graebe) Control System Design (Upper Saddle River, NJ: Prentice-Hall, 2001).

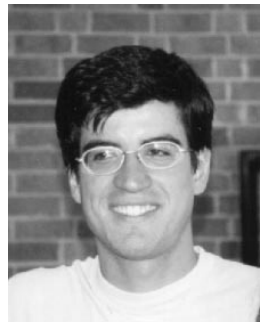

Juan I. Yuz was born in 1975. He received the professional title of Ingeniero Civil Electrónico and the M.S. degree in electronics engineering from Universidad Técnica Federico Santa María, Valparaíso, Chile, in 2001. He is currently working toward the $\mathrm{Ph} . \mathrm{D}$. degree in electrical engineering at The University of Newcastle, Newcastle, NSW, Australia.

His research areas include performance limitations, control with constraints, and system identification. 